\title{
Obstructive sleep apnoea is associated with decreased insulin sensitivity in females
}

\author{
J. Theorell-Haglöw*, C. Berne ${ }^{\#}$, C. Janson* and E. Lindberg*
}

ABSTRACT: The aim of the present study was to assess associations between obstructive sleep apnoea and insulin sensitivity in a population-based sample of females.

In total, 400 females aged 20-70 yrs underwent a full-night polysomnography, fasting blood sampling, measurement of anthropometric variables and oral glucose tolerance test with measurement of the insulin response $(n=358)$. The apnoea/hypopnoea index $(\mathrm{AHI})$ was calculated from the results of the polysomnography. From the results of the oral glucose tolerance test, an insulin sensitivity index (ISI) was calculated.

Females with an $\mathrm{AHI}<5(\mathrm{n}=119)$ had a mean \pm SD ISI of $8.3 \pm 3.8$, whereas females with an AHI $\geqslant 30(n=34)$ had an ISI of $6.2 \pm 4.0$. Nocturnal minimal saturation was independently associated with decreased insulin sensitivity when controlling for age, waist/hip ratio, level of physical activity, smoking and alcohol consumption (95\% confidence interval (Cl) 0.004-0.14). When adjusting for confounders, the AHI was associated with increased fasting and 2-h insulin levels (95\% Cl 0.14-0.99 and 95\% Cl 0.28-6.47, respectively).

Obstructive sleep apnoea was found to be independently associated with decreased insulin sensitivity in the present population-based sample of females.

KEYWORDS: Females, glucose metabolism, insulin sensitivity, population-based, sleep-disordered breathing

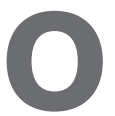
bstructive sleep apnoea (OSA) syndrome and diabetes mellitus are both closely related to obesity [1]. Studies have indicated an independent association between the two conditions, as treatment of OSA syndrome patients with continuous positive airway pressure (CPAP) results in an improvement in glucose metabolism [2-4], although there are also conflicting studies [5, 6]. Furthermore, in populationbased studies, an independent association between self-reported snoring, a common symptom of sleep-disordered breathing, and incident diabetes has been reported in both males [7] and females [8]. In nonobese males, self-reported snoring was also associated with decreased insulin sensitivity [9].

Studies on the impact of measured OSA, however, have focused primarily on clinical patients with OSA syndrome [2, 10-12]. The role of a sleep-related breathing disorder as a risk factor for diabetes mellitus in a general female population is less clear. Impaired insulin sensitivity is one of the major defects underlying the development of type 2 diabetes mellitus. The aim of the present study was therefore to assess the relationships between OSA and insulin sensitivity in a population-based sample of females.

\section{METHODS}

\section{Population}

The second phase of the population-based study "Sleep and Health in Women" was conducted between 2002 and 2004 [13]. In the first phase, randomly selected females (aged $\geqslant 20$ yrs) from the population registry of the city of Uppsala, Sweden, were sent a questionnaire on sleep disturbances and somatic disorders; 7,051 $(71.6 \%)$ of the females responded [13]. Figure 1 shows a flow chart of the study design. In the questionnaire, snoring was assessed by the question: "How often do you snore loudly and disturbingly?" The response options were as follows: "Never" (1), "Seldom" (2), "Sometimes" (3), "Often" (4) and "Very often" (5). Based on the females' response to this question, the participants were categorised as nonsnorers (scores 1-3; $\mathrm{n}=6,515$ ) and snorers (scores $4-5 ; \mathrm{n}=536$ ). In the second phase of the study, a sample of 400 females were selected from the responders in the first phase aged $<70 \mathrm{yrs}(\mathrm{n}=6,112)$. Of the 400 females, 230 were selected randomly from the snorers and 170 were selected randomly from the whole group. The sampling was conducted in this way in order to obtain an over-sampling of snorers. Subjects who
AFFILIATIONS

Depts of *Medical Sciences,

Respiratory Medicine and

Allergology, and

\#Internal Medicine, Uppsala

University, Uppsala, Sweden.

\section{CORRESPONDENCE}

J. Theorell-Haglöw

Dept of Medical Sciences,

Respiratory Medicine and Allergology

Uppsala University

Akademiska sjukhuset

SE-751 85 Uppsala

Sweden

Fax: 46186110228

E-mail: jenny.theorell-haglow@

medsci.uu.se

Received:

June 202007

Accepted after revision:

December 172007

SUPPORT STATEMENT

This study was financially supported

by the Swedish Heart Lung

Foundation.

STATEMENT OF INTEREST

None declared. 
were expected to be unable to carry out the ambulatory recordings due to severe somatic or psychiatric disease were excluded.

\section{Polysomnography}

All females underwent whole-night polysomnography either in their own home or at the hotel associated with the hospital, using the ambulatory system EMBLA (Flaga Inc., Reykjavik, Iceland). Sixteen channels were recorded and included two electro-encephalography leads (C3-A2 and C4-A1), two electrooculography leads and three electromyography leads (submental, and left and right anterior tibialis muscles). In addition, two airflow leads (oronasal thermistor and nasal flow pressure sensor), one pharyngeal sound lead (piezo vibration sensor) and two respiratory effort leads (thoracic and abdominal piezo crystal transducers) were included. Furthermore, one oxymeter lead (to measure oxygen saturation level and pulse from a finger probe), two electrocardiography leads and one body position lead were included. Data were downloaded to the Somnologica reviewing analysis software (Version 2.0; Flaga Inc.) and sleep was scored manually in 30-s epochs according to standard criteria [14]. The polysomnography was considered acceptable when $\geqslant 4$ h of sleep were recorded and no registration had been lost for $\geqslant 20 \mathrm{~min}$ of the night. A total of six polysomnography recordings had to be redone due to poor quality of the first recording. An apnoea was defined as the complete cessation of nasal and oral airflow lasting $\geqslant 10 \mathrm{~s}$. Hypopnoea was defined as $a \geqslant 50 \%$ reduction in airflow amplitude compared with baseline, in combination with a reduction of $\geqslant 3 \%$ in oxyhaemoglobin

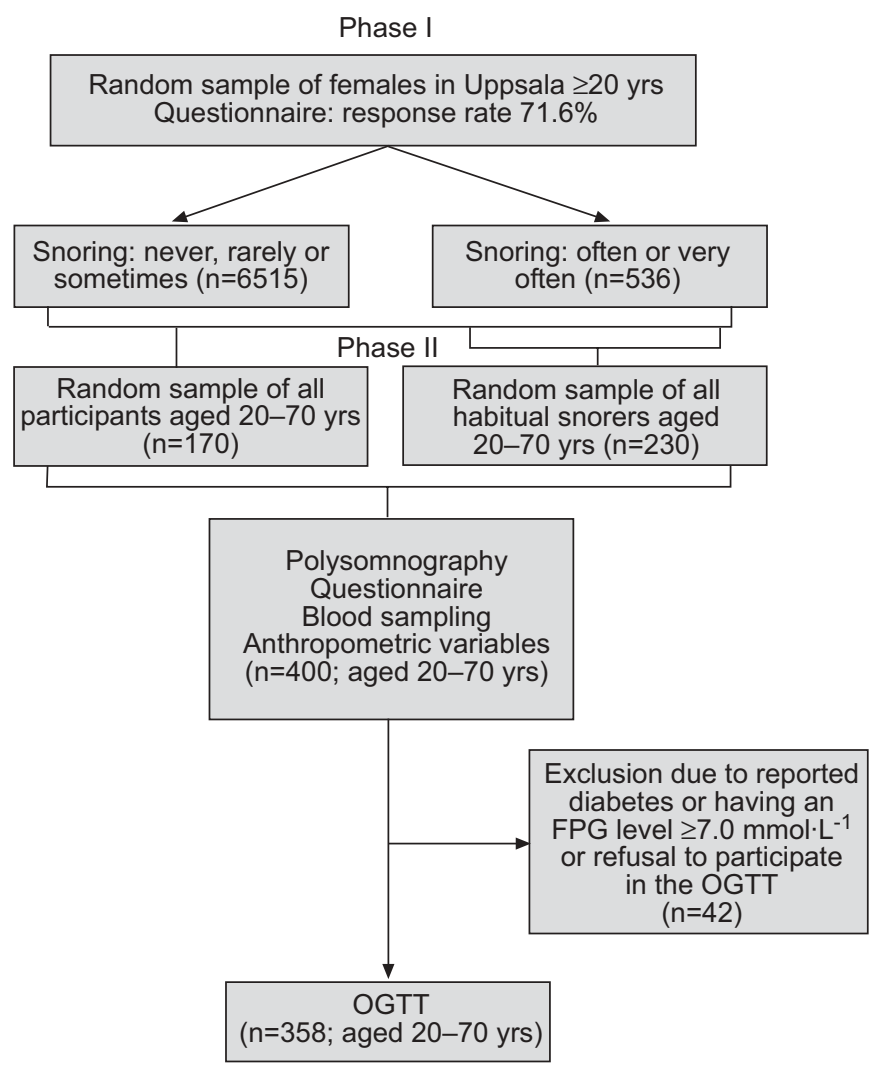

FIGURE 1. Flow chart of the present study's design. FPG: fasting plasma glucose; OGTT: oral glucose tolerance test. saturation, or an arousal. An apnoea/hypopnoea index (AHI; the average number of apnoeas and hypopnoeas per hour of sleep) was calculated.

\section{Blood sampling and anthropometric variables}

In the morning following the polysomnography, the females returned fasting to the laboratory. Venous blood samples were taken for analyses of plasma glucose, serum insulin and haemoglobin A1c.

Anthropometric variables (height, weight, waist circumference and hip circumference) were measured by a research nurse. Body mass index (BMI) was calculated as weight $(\mathrm{kg}) /$ height $^{2}$ $\left(\mathrm{m}^{2}\right)$ and then categorised into three groups: $<20,20-<25$ and $\geqslant 25 \mathrm{~kg} \cdot \mathrm{m}^{-2}$. Waist circumference was measured midway between the lower rib margin and the anterior superior iliac spine; hip circumference was measured at the widest circumference over the great trochanters; the waist/hip ratio (WHR) was calculated. Blood pressure was measured in the right arm after a $15 \mathrm{~min}$ rest in a supine position.

\section{Questionnaires}

Prior to the polysomnography, the female subjects filled in questionnaires, including questions on somatic disease, medication, snoring, daytime sleepiness, physical activity, tobacco use and alcohol consumption.

The participants' physical activity was analysed by four questions adopted from a questionnaire used in a large population-based study on the correlation of physical activity and mortality in females [15]. The females were asked to respond on a scale of $1-4$, where $1=$ spending most leisure time at sedentary activities, $2=$ exercising for $\geqslant 4 \mathrm{~h}$ per week (walking, cycling back and forth to work, etc.), $3=\geqslant 3 \mathrm{~h}$ of keep-fit exercises per week, and $4=$ hard exercise several times a week. This gave the following three categories of physical activity: low (score 1), medium (score 2) and high activity (scores 3-4).

Six questions assessed smoking habits [16]. Based on the participants' responses, they were categorised as either "current smokers" or "nonsmokers" (i.e. never smoked or had quit smoking $\geqslant 6$ months before answering the questionnaire).

To investigate alcohol consumption, the females were asked to state how many millilitres of different kinds of alcoholic beverages they drank per week. From this information, the total amount of alcohol in $\mathrm{g} \cdot \mathrm{week}^{-1}$ was calculated using the following formula:

$(($ volume percentage alcohol $(\%) \times$ total volume $(\mathrm{mL})) / 100) \times 0.7894$

where 0.7894 is the density of the alcohol in $\mathrm{g} \cdot \mathrm{L}^{-1}$.

\section{Oral glucose tolerance test}

Within $\sim 1$ month of the polysomnography (mean latency period, 36 days), the females underwent an oral glucose tolerance test (OGTT). Exclusion criteria for performing the OGTT were stating diabetes mellitus or medication for diabetes mellitus in the questionnaires. In addition, females with a fasting plasma glucose (FPG) level $\geqslant 7.0 \mathrm{mmol} \cdot \mathrm{L}^{-1}$ at the time of the OGTT or females who refused to participate in the OGTT were excluded. The OGTT was performed in 358 of 
the females (fig. 1). The test was conducted by administration of $75 \mathrm{~g}$ of glucose, and venous blood samples of plasma glucose and serum insulin were taken at 0,30, 60, 90 and $120 \mathrm{~min}$. Type 2 diabetes mellitus was defined according to World Health Organization $(\mathrm{WHO})$ criteria as $\mathrm{FPG} \geqslant 7.0 \mathrm{mmol} \cdot \mathrm{L}^{-1}$ or 2 -h postglucose load $\geqslant 11.1 \mathrm{mmol} \cdot \mathrm{L}^{-1}$. Impaired fasting glucose (IFG) was defined as FPG 6.1-7.0 $\mathrm{mmol} \cdot \mathrm{L}^{-1}$ with 2 -h post-glucose load $<7.8 \mathrm{mmol} \cdot \mathrm{L}^{-1}$ [17]. Impaired glucose tolerance was defined as FPG $<7.0 \mathrm{mmol} \cdot \mathrm{L}^{-1}$ with 2 -h post-glucose load 7.8 $11.1 \mathrm{mmol} \cdot \mathrm{L}^{-1}$ [17]. Impaired glucose metabolism was defined as stating type 2 diabetes mellitus in the questionnaires or having IFG, impaired glucose tolerance or type 2 diabetes mellitus according to the WHO criteria [17]. Insulin sensitivity was calculated as an index (insulin sensitivity index (ISI)) based on FPG, fasting plasma insulin (FPI), mean OGTT glucose concentration and mean OGTT insulin concentration. The formula for ISI was as follows:

$10,000 /(($ FPG $\times$ FPI $\times$ mean OGTT glucose

concentration $\times$ mean OGTT insulin concentration) $)^{0.5}$ )

This index has been shown to provide a good estimate of whole-body insulin sensitivity and correlates well with the euglycaemic insulin clamp [18].

\section{Statistical analyses}

Univariate analyses were conducted using the unpaired t-test or the Chi-squared test to compare baseline data between groups. Associations between variables of OSA and glucose metabolism were analysed using multiple regression analysis. As there was a close relationship between BMI and WHR $(\mathrm{r}=0.34 ; \mathrm{p}<0.0001)$, only WHR was used in the multiple regression analysis. Results from the regression analysis are presented as $\beta$-values with $95 \%$ confidence intervals. Furthermore, interaction analyses were conducted to detect significant differences in associations between OSA and insulin sensitivity in older $(\geqslant 50 \mathrm{yrs})$ and younger females ( $<50 \mathrm{yrs})$. To detect differences between females in different $\mathrm{AHI}$ groups from the OGTT, ANOVA analyses were performed using results from the five measure points of the OGTT. A p-value $<0.05$ was considered to indicate a significant difference.

The present study was approved by the Ethics Committee of the Medical Faculty at Uppsala University and all participants in the study gave their informed consent.

\section{RESULTS}

Table 1 shows the characteristics of the females with different levels of AHI. Females with an AHI of $\geqslant 5$ (i.e. females with OSA) had significantly higher mean FPG and mean fasting serum insulin compared with females with AHI $<5$. In addition, the ISI was significantly lower in females with OSA than in females without, and the females with OSA were also generally older, more overweight and more hypertensive. There was no significant difference in total sleep time between the groups of females; however, females with an AHI of $\geqslant 15$

TABLE 1 Characteristics of females in different obstructive sleep apnoea groups

\begin{tabular}{|c|c|c|c|c|c|c|c|}
\hline & $\mathrm{AHI}<5$ & AHI 5-15 & p-value ${ }^{\#}$ & AHI 15-30 & p-value ${ }^{\#}$ & $A H I \geqslant 30$ & p-value ${ }^{\#}$ \\
\hline Subjects $n$ & 134 & 131 & & 93 & & 42 & \\
\hline Mean saturation \% & $96.0 \pm 1.60$ & $95.3 \pm 1.7$ & 0.0006 & $94.8 \pm 1.4$ & $<0.0001$ & $93.9 \pm 1.8$ & $<0.0001$ \\
\hline Minimal saturation \% & $91.1 \pm 4.8$ & $88.0 \pm 5.4$ & $<0.0001$ & $85.2 \pm 5.1$ & $<0.0001$ & $81.1 \pm 6.5$ & $<0.0001$ \\
\hline Age yrs & $43.5 \pm 11.4$ & $50.9 \pm 9.9$ & $<0.0001$ & $54.8 \pm 9.6$ & $<0.0001$ & $58.0 \pm 6.3$ & $<0.0001$ \\
\hline Stages 3 and 4 sleep $\%$ & $10.6 \pm 5.9$ & $9.3 \pm 5.4$ & 0.07 & $9.6 \pm 5.8$ & 0.21 & $7.7 \pm 5.9$ & 0.006 \\
\hline REM sleep \% & $19.0 \pm 6.0$ & $18.8 \pm 8.22$ & 0.81 & $17.2 \pm 6.1$ & 0.03 & $14.6 \pm 7.1$ & 0.0001 \\
\hline$B M I \mathbf{k g} \cdot \mathrm{m}^{-2}$ & $24.7 \pm 3.7$ & $26.5 \pm 4.8$ & 0.0005 & $27.8 \pm 5.1$ & $<0.0001$ & $30.8 \pm 5.8$ & $<0.0001$ \\
\hline WHR & $0.83 \pm 0.08$ & $0.85 \pm 0.06$ & 0.04 & $0.87 \pm 0.06$ & 0.0002 & $0.90 \pm 0.8$ & $<0.0001$ \\
\hline Plasma glucose $\mathrm{mmol} \cdot \mathrm{L}^{-1}$ & $5.2 \pm 0.83$ & $5.5 \pm 1.2$ & 0.02 & $5.5 \pm 0.80$ & 0.03 & $6.1 \pm 1.7$ & $<0.0001$ \\
\hline Serum insulin $\mathrm{mU} \cdot \mathrm{L}^{-1}$ & $6.4 \pm 3.5$ & $7.5 \pm 6.6$ & 0.09 & $8.8 \pm 6.3$ & 0.0004 & $10.1 \pm 6.7$ & $<0.0001$ \\
\hline Smoker & 29 (21.6) & $23(17.8)$ & 0.44 & $21(23.1)$ & 0.80 & 11 (28.2) & 0.44 \\
\hline Physical activity & & & 0.50 & & 0.17 & & 0.16 \\
\hline High & 29 (21.8) & $22(17.1)$ & & $11(12.2)$ & & $4(10.0)$ & \\
\hline Medium & $90(67.7)$ & $89(69.0)$ & & $70(77.8)$ & & 29 (72.5) & \\
\hline Low & $14(10.5)$ & $18(14.0)$ & & $9(10.0)$ & & 7 (17.5) & \\
\hline Alcohol g. week ${ }^{-1}$ & $54.1 \pm 66.1$ & $56.3 \pm 52.2$ & 0.77 & $54.2 \pm 52.6$ & 0.85 & $57.1 \pm 62.9$ & 0.73 \\
\hline
\end{tabular}

Data are presented as mean \pm SD or $n(\%)$, unless otherwise indicated. AHI: apnoea/hypopnoea index; ODI: oxygen desaturation index; REM: rapid eye movement; BMI: body mass index; WHR: waist/hip ratio; ISI: insulin sensitivity index. ${ }^{*}$ : p-values are calculated using females with AHI <5 as control; ${ }^{\bullet}$ : having impaired fasting glucose or impaired glucose tolerance or type 2 diabetes mellitus; ${ }^{+}$: systolic blood pressure $\geqslant 130 \mathrm{mmHg}$ and/or diastolic blood pressure $\geqslant 90 \mathrm{mmHg}$ or known hypertension or being medicated for hypertension. 
had less rapid eye movement sleep compared with females without OSA. Furthermore, the females in the highest AHI group showed a significantly lower percentage of stages 3 and 4 sleep compared with the females without OSA (table 1).

The results from the OGTT are depicted in figure 2. There were significant differences between the plasma glucose curve for females in different AHI groups throughout the OGTT, showing a dose-response relationship between $\mathrm{AHI}$ and plasma glucose. Furthermore, there was an inverse relationship between AHI and the disappearance of plasma glucose. In addition, a dose-response relationship was seen between AHI and serum insulin throughout the insulin curve (fig. 2b). Results from the OGTT were also analysed in females with a BMI $<27 \mathrm{~kg} \cdot \mathrm{m}^{-2}$ (population mean), but these results did not differ from those of the whole group (data not shown).

When calculating ISI in this population of females, $n=352$ because, in order to calculate ISI, there must be no missing values for any of the samples throughout the OGTT. For six of the females who underwent the OGTT, one value was missing
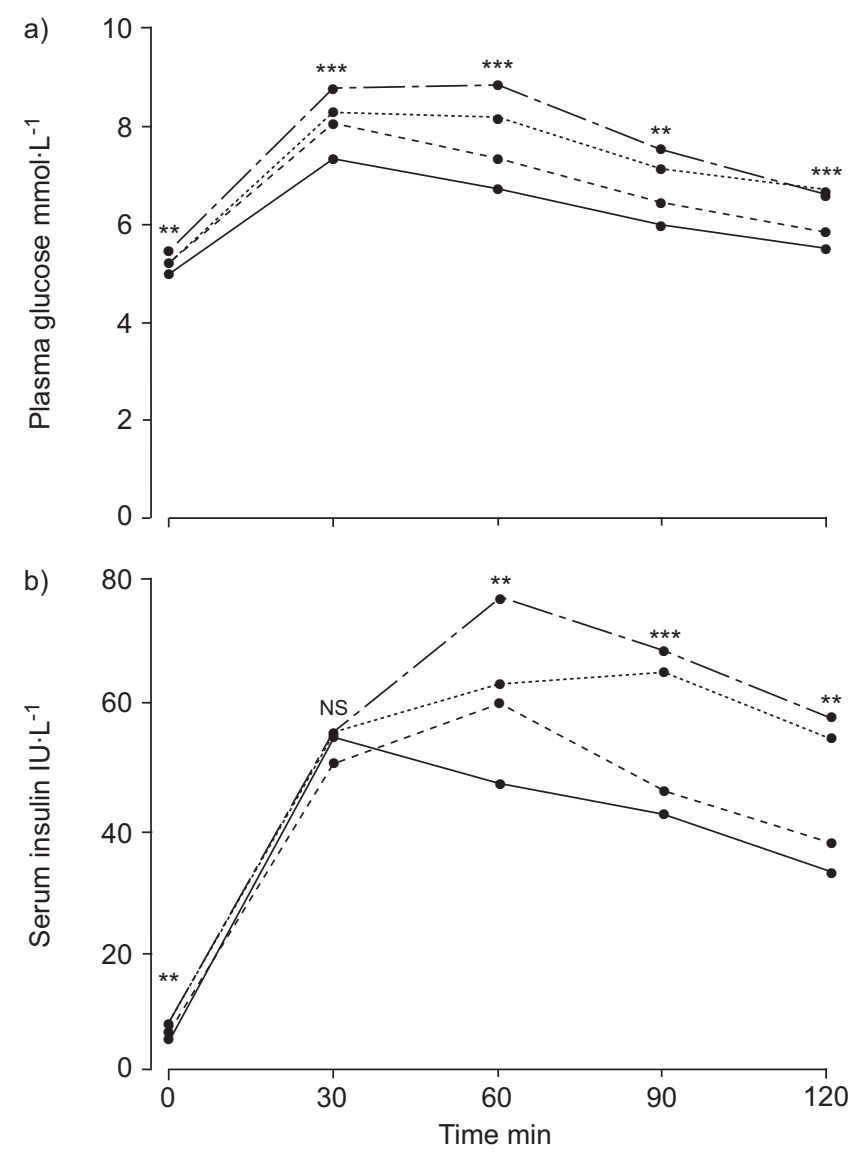

FIGURE 2. a) Plasma glucose concentrations during the oral glucose tolerance test (OGTT) in females with different apnoea/hypopnoea indices (AHI). b) Serum insulin concentrations during the OGTT in females with different AHI. Using ANOVA, the $\mathrm{p}$-values were calculated for differences between AHI groups at the five points of measurement $(0,30,60,90$ and $120 \mathrm{~min})$ in the OGTT. NS: nonsignificant. —- $\mathrm{AHI}<5(\mathrm{n}=120) ;-----$ : AHI $5-<15(n=115) ; \cdots \cdots \cdots \cdot$ AHI $15-<30(n=88) ;-----: A H I \geqslant 30(n=35) . * *$ : $p$-value between groups $<0.01 ; * * *$ : p-value between groups $<0.0001$. and therefore the ISI could not be calculated, thus giving a total number of subjects of $n=352$. There was a gradual decrease in ISI with increasing AHI, i.e. females with $\mathrm{AHI}<5$ showed a mean \pm SD ISI of $8.3 \pm 3.8$, whereas females with $\mathrm{AHI} \geqslant 30$ had an ISI of $6.2 \pm 4.0$ (p-value for trend $<0.0001$; fig. 3 ). Furthermore, the prevalence of impaired glucose metabolism increased with $\mathrm{AHI}$ from $9 \%$ of the females with $\mathrm{AHI}<5$ to $42.9 \%$ in females with AHI $\geqslant 30$ ( $\mathrm{p}$-value for trend $<0.0001$; fig. 3 ).

\section{Multivariate analysis}

Multiple linear regression analysis was performed to assess the relationship between variables of OSA and insulin sensitivity (table 2). When controlling for confounders (age, WHR, level of physical activity, smoking and alcohol consumption) a low nocturnal minimal saturation was significantly correlated with decreased ISI. Moreover, low minimal saturation during the night was associated with higher plasma glucose and serum insulin concentrations at the end of the OGTT (2 h). AHI was independently associated with fasting serum insulin concentration and increased concentrations of 2-h serum insulin at the OGTT. The percentage of time during the night with saturation $<90 \%$ was independently associated with fasting serum insulin levels, whereas the association with the ISI did not reach statistical significance. However, in the 236 females $\geqslant 50$ yrs there was a significant association between percentage of night with saturation $<90 \%$ and ISI (table 3 ).

\section{DISCUSSION}

The main observation from the present study is that there is an association between OSA and insulin sensitivity in a population-based sample of females. This association is independent of age, WHR, level of physical activity, smoking and alcohol consumption. Although the relationship between OSA

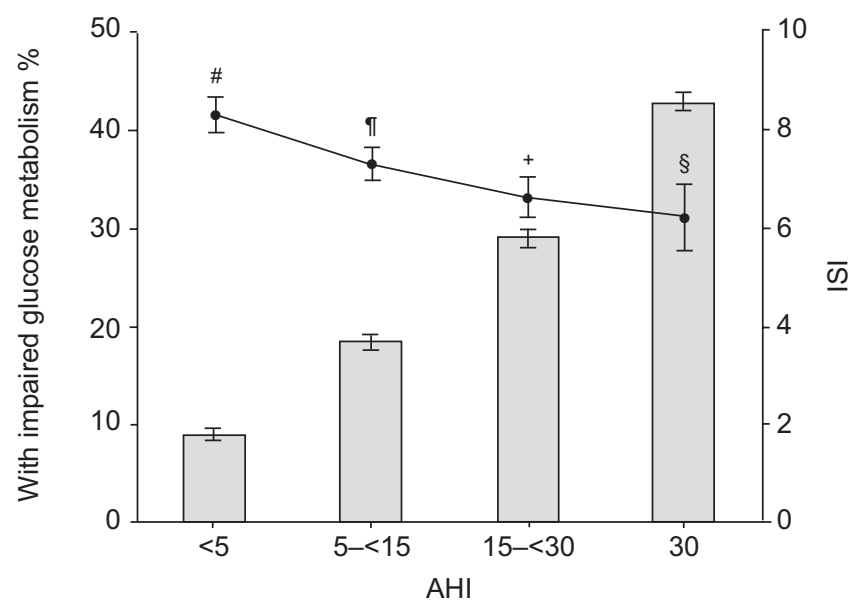

FIGURE 3. Prevalence of impaired glucose metabolism and mean insulin sensitivity index (ISI) in females of different apnoea/hypopnoea index (AHI) groups. When calculating ISI, no missing values for any of the samples throughout the oral glucose tolerance test are allowed. Therefore, the number of females in different $\mathrm{AHI}$ groups is lower for ISI than for impaired glucose metabolism. The number of subjects in each $\mathrm{AHI}$ group was as follows: $\mathrm{AHI}<5: \mathrm{n}=134$; $\mathrm{AHI} 5-<15: n=131$; $\mathrm{AHI} 15-<30: n=93 ; \mathrm{AHI} 30: \mathrm{n}=42$. $\quad$ : the prevalence of impaired glucose metabolism in per cent is shown with $95 \%$ confidence intervals. $\bullet$ : ISI mean \pm SE.

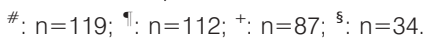




\begin{tabular}{|c|c|c|c|c|c|c|}
\hline \multirow[t]{2}{*}{ TABLE 2} & \multicolumn{6}{|c|}{$\begin{array}{l}\text { Associations between variables of obstructive sleep apnoea and glucose metabolism obtained at the oral glucose } \\
\text { tolerance test }\end{array}$} \\
\hline & & $\begin{array}{l}\text { Fasting plasma glucose } \\
\qquad \mathrm{mmol} \cdot \mathrm{L}^{-1}\end{array}$ & $\begin{array}{l}\text { Fasting serum insulin } \\
\qquad \mathrm{IU} \cdot \mathrm{L}^{-1}\end{array}$ & $\begin{array}{l}\text { Plasma glucose at } \\
120 \mathrm{~min} \mathrm{mmol} \cdot \mathrm{L}^{-1}\end{array}$ & $\begin{array}{l}\text { Serum insulin at } \\
120 \mathrm{~min} \mathrm{IU} \cdot \mathrm{L}^{-1}\end{array}$ & ISI \\
\hline \multicolumn{2}{|l|}{$\mathrm{AHI} \mathrm{I}^{\#}$} & $0.13(-0.07-0.09)$ & $0.56(0.14-0.99)$ & $0.024(-0.12-0.17)$ & $3.38(0.28-6.47)$ & $-0.12(-0.41-0.17)$ \\
\hline \multicolumn{2}{|c|}{ ODI } & $-0.012(-0.10-0.07)$ & $0.37(-0.08-0.83)$ & $-0.007(-0.16-0.15)$ & $1.81(-1.43-5.06)$ & $-0.18(-0.48-0.12)$ \\
\hline \multicolumn{2}{|c|}{$\begin{array}{l}\% \text { of the night with } \\
\text { saturation }<\mathbf{9 0 \%}\end{array}$} & $0.014(-0.004-0.03)$ & $0.10(0.001-0.20)$ & $0.026(-0.13-0.07)$ & $0.098(-0.74-0.94)$ & $-0.04(-0.12-0.04)$ \\
\hline
\end{tabular}

Results are presented as $\beta$-values (95\% confidence interval) from multiple regression analysis adjusting for age, waist/hip ratio, level of activity, smoking and alcohol consumption. ISI: insulin sensitivity index; AHI: apnoea/hypopnoea index; ODI: oxygen desaturation index. ${ }^{\#}$ : results are calculated for an increase of 10 units.

and insulin sensitivity can partly be explained by shared risk factors, AHI was found to be independently associated with increased fasting serum insulin. In addition, low nocturnal minimal saturation was independently associated with decreased insulin sensitivity. AHI and measurements of hypoxia were independently associated with the late serum insulin response $2 \mathrm{~h}$ after ingestion of $75 \mathrm{~g}$ of glucose.

The dose-response relationship between the AHI level and plasma glucose and serum insulin concentrations indicates that OSA may, as it worsens and AHI increases, gradually affect glucose metabolism and impair insulin sensitivity. A similar dose-response relationship has recently been reported in patients with OSA syndrome [19, 20]. Independent associations between OSA and measures of glucose metabolism have also been reported in a population-based study of males [21] and in males with OSA syndrome [12]. The negative impact of sleep-disordered breathing on glucose metabolism is further supported in a prospective study by the observation that snorers have an increased risk of developing diabetes [7]. Using either a doctor's diagnosis or blood sampling results for diagnosis of diabetes, longitudinal data from the Wisconsin Sleep Cohort show that diabetes is more prevalent in sleepdisordered breathing, and that this association is independent of other risk factors [22].

Subjects with OSA often exhibit coexisting risk factors (i.e. central obesity, dyslipidaemia, hyperglycaemia and hypertension) for cardiovascular disease and diabetes. In the present study, however, AHI and measurements of hypoxia were associated with changes in glucose metabolism, independent of age, central obesity, level of physical activity, smoking and alcohol consumption. These findings indicate that OSA decreases insulin sensitivity and may contribute to an increased risk of developing diabetes independently of central obesity. In addition, low minimal saturation was independently associated with a low ISI, suggesting that the negative influence of sleep-disordered breathing on insulin sensitivity may be mediated though hypoxic pathways. Furthermore, in several studies, $\mathrm{AHI}$ and hypoxia have been related to glucose dysregulation and insulin resistance [11, 20, 23]. The present study also showed that in older females, the association between hypoxia and insulin sensitivity was stronger, a result indicating that age may increase the sensitivity to hypoxia.

OSA can affect glucose metabolism and insulin sensitivity negatively through several pathways. First, lack of sleep influences metabolic and endocrine function with alterations of glucose and insulin profiles as a result, which decreases glucose tolerance [24]. Sleep loss has also been suggested to increase levels of cortisol through increased activity in the hypothalamic-pituitary-adrenal axis [25], a mechanism that can contribute to hyperglycaemia. Secondly, the intermittent hypoxaemia and reoxygenation accompanying obstructive apnoeas may trigger the formation of inflammatory cytokines [26], which promotes peripheral insulin resistance [27]. VGONTZAS et al. [28] found higher levels of the inflammatory cytokines

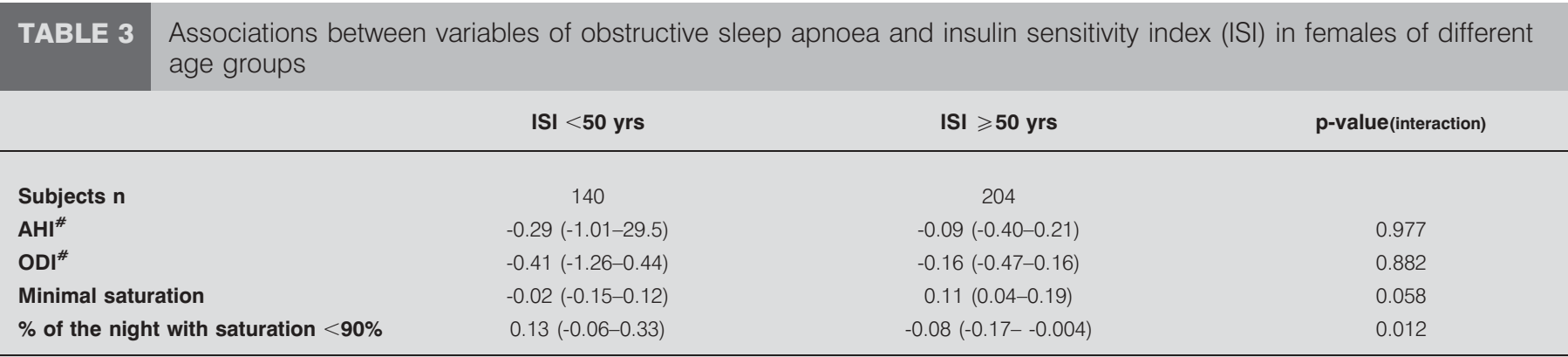


tumour necrosis factor- $\alpha$ and interleukin- 6 in patients with OSA syndrome compared with weight-matched controls without OSA syndrome. In addition, the deoxygenation and reoxygenation cycles in OSA provide an environment with increased oxidative stress [26, 29], which, in combination with hyperglycaemia, may promote the formation of advanced glycation end-products [30]. Advanced glycation end-products are implicated in the progression of micro- and macrovascular complications of diabetes [31]. Finally, arousals accompanying obstructive apnoea increase nocturnal sympathetic activity, and increased urinary and plasma catecholamines are displayed in OSA patients [32] Sympathetic hyperactivity may also in turn negatively affect glucose homeostasis by enhancing hepatic glucose production and inducing skeletal muscle insulin resistance.

Effectively treating obstructive apnoeas, which reduces sleep loss, hypoxia and sympathetic hyperactivity in females with OSA, may promote increased insulin sensitivity and reduce the risk of diabetes mellitus. Some studies show improved insulin sensitivity when patients with both diabetes and OSA syndrome are treated with CPAP $[2,3,10]$. However, two recent studies have not shown improvement in insulin sensitivity with CPAP $[5,6]$. In both these studies, the majority or all of the subjects were obese, which may have had an impact on the outcome. In an earlier study by HARSCH et al. [2], the improvement in insulin sensitivity by CPAP was smaller in the obese than in the nonobese patients. HARSCH et al. [2] suggested that insulin sensitivity in obese individuals is mainly determined by obesity and, to a smaller extent, by sleep apnoea [2].

The present study was conducted in a large population-based sample of females in which relevant objective data on OSA and glucose metabolism were collected. However, there are some considerations that should be borne in mind when interpreting the results of the present study. The use of the ISI as a surrogate from the OGTT instead of the gold standard euglycaemic insulin clamp when assessing insulin sensitivity may be a less precise measure of insulin sensitivity. Nevertheless, the ISI used in the present study has been shown to provide a good estimate $(r=0.73, \mathrm{p}<0.0001)$ of whole-body insulin sensitivity when compared with the euglycaemic insulin clamp [18].

In summary, in a population-based sample of females, several features of obstructive sleep apnoea displayed independent associations with decreased insulin sensitivity. This indicates that decreased insulin sensitivity should be considered when treating patients with obstructive sleep apnoea.

\section{ACKNOWLEDGEMENTS}

The authors would like to thank C. Sahlin (Dept of Respiratory Medicine, Umeå University Hospital, Umeå, Sweden) for her assistance with the polysomnography scoring in this study.

\section{REFERENCES}

1 Young T, Peppard PE, Gottlieb DJ. Epidemiology of obstructive sleep apnea: a population health perspective. Am J Respir Crit Care Med 2002; 165: 1217-1239.

2 Harsch IA, Schahin SP, Radespiel-Tröger M, et al. Continuous positive airway pressure treatment rapidly improves insulin sensitivity in patients with obstructive sleep apnea syndrome. Am J Respir Crit Care Med 2004; 169: 156-162.

3 Babu AR, Herdegen J, Fogelfeld L, Shott S, Mazzone T. Type 2 diabetes, glycemic control, and continuous positive airway pressure in obstructive sleep apnea. Arch Intern Med 2005; 165: 447-452.

4 Lindberg E, Berne C, Elmasry A, Hedner J, Janson C. CPAP treatment of a population-based sample-what are the benefits and the treatment compliance? Sleep Med 2006; 7: 553-560.

5 Coughlin SR, Mawdsley L, Mugarza JA, Wilding JP, Calverley PM. Cardiovascular and metabolic effects of CPAP in obese males with OSA. Eur Respir J 2007; 29: 720-727.

6 West SD, Nicoll DJ, Wallace TM, Matthews DR, Stradling JR. Effect of CPAP on insulin resistance and $\mathrm{HbA1c}$ in men with obstructive sleep apnoea and type 2 diabetes. Thorax 2007; 62: 969-974.

7 Elmasry A, Janson C, Lindberg E, Gislason T, Tageldin MA, Boman G. The role of habitual snoring and obesity in the development of diabetes: a 10-year follow-up study in a male population. J Intern Med 2000; 248: 13-20.

8 Al-Delaimy WK, Manson JE, Willett WC, Stampfer MJ, $\mathrm{Hu}$ FB. Snoring as a risk factor for type II diabetes mellitus: a prospective study. Am J Epidemiol 2002; 155: 387-393.

9 Shin C, Kim J, Kim J, et al. Association of habitual snoring with glucose and insulin metabolism in nonobese Korean adult men. Am J Respir Crit Care Med 2005; 171: 287-291.

10 Czupryniak L, Loba J, Pawlowski M, Nowak D, Bialasiewicz P. Treatment with continuous positive airway pressure may affect blood glucose levels in nondiabetic patients with obstructive sleep apnea syndrome. Sleep 2005; 28: 601-603.

11 Ip MS, Lam B, Ng MM, Lam WK, Tsang KW, Lam KS. Obstructive sleep apnea is independently associated with insulin resistance. Am J Respir Crit Care Med 2002; 165: 670-676.

12 Meslier N, Gagnadoux F, Giraud P, et al. Impaired glucoseinsulin metabolism in males with obstructive sleep apnoea syndrome. Eur Respir J 2003; 22: 156-160.

13 Theorell-Haglöw J, Lindberg E, Janson C. What are the important risk factors for daytime sleepiness and fatigue in women? Sleep 2006; 29: 751-757.

14 Rechtschaffen A, Kales A, eds. A Manual of Standardized Terminology, Techniques, and Scoring System for Sleep Stages in Human Subjects. Washington DC, US National Public Health Service, US Government Printing Office, 1968.

15 Lissner L, Bengtsson C, Björkelund C, Wedel H. Physical activity levels and changes in relation to longevity. A prospective study of Swedish women. Am J Epidemiol 1996; 143: 54-62.

16 Svensson M, Lindberg E, Naessen T, Janson C. Risk factors associated with snoring in women with special emphasis on body mass index: a population-based study. Chest 2006; 129: 933-941.

17 Definition, Diagnosis and Classification of Diabetes Mellitus and its Complications. Report of a WHO Consultation. Part 1: Diagnosis and Classification of Diabetes Mellitus. Geneva, World Health Organization, 1999; p. 59.

18 Matsuda M, DeFronzo RA. Insulin sensitivity indices obtained from oral glucose tolerance testing: comparison 
with the euglycemic insulin clamp. Diabetes Care 1999; 22: 1462-1470.

19 Makino S, Handa H, Suzukawa K, et al. Obstructive sleep apnoea syndrome, plasma adiponectin levels, and insulin resistance. Clin Endocrinol (Oxford) 2006; 64: 12-19.

20 Punjabi NM, Sorkin JD, Katzel LI, Goldberg AP, Schwartz AR, Smith PL. Sleep-disordered breathing and insulin resistance in middle-aged and overweight men. Am J Respir Crit Care Med 2002; 165: 677-682.

21 Elmasry A, Lindberg E, Berne C, et al. Sleep-disordered breathing and glucose metabolism in hypertensive men: a population-based study. J Intern Med 2001; 249: 153-161.

22 Reichmuth KJ, Austin D, Skatrud JB, Young T. Association of sleep apnea and type II diabetes: a population-based study. Am J Respir Crit Care Med 2005; 172: 1590-1595.

23 Punjabi NM, Shahar E, Redline S, et al. Sleep-disordered breathing, glucose intolerance, and insulin resistance: the Sleep Heart Health Study. Am J Epidemiol 2004; 160: 521-530.

24 Spiegel K, Leproult R, L'hermite-Balériaux M, Copinschi G, Penev PD, Van Cauter E. Leptin levels are dependent on sleep duration: relationships with sympathovagal balance, carbohydrate regulation, cortisol, and thyrotropin. J Clin Endocrinol Metab 2004; 89: 5762-5771.
25 Spiegel K, Leproult R, Van Cauter E. Impact of sleep debt on metabolic and endocrine function. Lancet 1999; 354: 1435-1439.

26 Dyugovskaya L, Lavie P, Lavie L. Increased adhesion molecules expression and production of reactive oxygen species in leukocytes of sleep apnea patients. Am J Respir Crit Care Med 2002; 165: 934-939.

27 Lyngso D, Simonsen L, Bulow J. Metabolic effects of interleukin-6 in human splanchnic and adipose tissue. $J$ Physiol 2002; 543: 379-386.

28 Vgontzas AN, Papanicolaou DA, Bixler EO, et al. Sleep apnea and daytime sleepiness and fatigue: relation to visceral obesity, insulin resistance, and hypercytokinemia. J Clin Endocrinol Metab 2000; 85: 1151-1158.

29 Tan KC, Chow WS, Lam JC, et al. Advanced glycation endproducts in nondiabetic patients with obstructive sleep apnea. Sleep 2006; 29: 329-333.

30 Singh R, Barden A, Mori T, Beilin L. Advanced glycation end-products: a review. Diabetologia 2001; 44: 129-146.

31 Brownlee $\mathrm{M}$. The pathobiology of diabetic complications: a unifying mechanism. Diabetes 2005; 54: 1615-1625.

32 Waradekar NV, Sinoway LI, Zwillich CW, Leuenberger UA. Influence of treatment on muscle sympathetic nerve activity in sleep apnea. Am J Respir Crit Care Med 1996; 153: 1333-1338. 